\title{
Surveillance with Alert Management System using Conventional Cell Phones
}

\author{
Tomas Brezmes, Marc Rersa \\ France Telecom R\&D, Spain \\ Barcelona, Spain \\ tomas.brezmes@ orange-ftgroup.com \\ marc.rersa@orange-ftgroup.com
}

\author{
Juan-Luis Gorricho, Josep Cotrina \\ Departamento de Telemática, \\ UPC Universitat Politècnica de Catalunya \\ Barcelona, Spain \\ juanluis@entel.upc.edu \\ jcotrina@entel.upc.edu
}

\begin{abstract}
In the present paper we expose the development of a real-time monitoring prototype of human activities using a single cell phone equipped with some sensors. The monitoring system is reduced to the minimum expression; no special hardware is required as far as a conventional cell phone will monitor the user, classify the user activities with respect to a customized rule's set, and finally trigger the corresponding alerts, when necessary.
\end{abstract}

Keywords-Pattern recognition; human movement's detection; cell phone's sensors; indoor location service.

\section{INTRODUCTION}

User activity monitoring has a considerable potential for a whole range of new applications in different environments: user surveillance, military purposes, health-care, etc. More precisely, in health-care there is an implicit potential application monitoring elderly people or patients suffering an illness. Besides, nowadays our society is more than ever exposed to an aging population, which is one of the main concerns of present administrations. Any technical approach alleviating the ever increasing health-care budget will be welcomed.

Thankfully, recent progress in communication technologies and sensor miniaturization has opened the door to new approaches overcoming the above mentioned situation, involving, for example, remote supervision of home-confined elderly people. In particular, a real-time monitoring system of human activities would be an interesting solution to supervise aged people or people deserving medical attention.

The aim of this study was to continue from previously developed prototypes on indoor location and movement recognition to achieve a deeper knowledge on the possibilities of context awareness to work out future services. In this respect, considering the relevance of pattern recognition on both indoor location and movement classification, one of the main efforts has been devoted to study and deploy several techniques focused on solving this matter, in particular, neural networks and support vector machines as the most promising candidates.

In order to put in practice any mathematical development, a representative prototype has been identified and implemented to carry out the validation tests and also to demonstrate the achieved results. In any case, the key issue for the intended scenario was to work out a representative prototype using a mobile phone with suitable enablers (wi-fi receiver, accelerometer, etc.) to obtain the input data which would lead us to the implementation of the context awareness paradigm.

\section{RELATED WORK}

Nowadays, common health-care monitoring appliances focus on strictly monitoring physiological parameters such as: the heart-beat pulse, blood pressure, breath activity, etc. using dedicated hospital equipment. But recently another type of health-care monitoring system is beginning to be envisaged as a useful tool for elderly people, in this case only focusing on monitoring the user activity [1] - [6]. These new appliances are presently in an early stage of development and they commonly operate on a 3 layer paradigm based on the following approach:

- A sensor layer: gathering relevant information on human activity. It uses some dedicated sensor devices embedding for example: accelerometers, gyroscopes, magnetometers, etc.

- A transmission layer: transmitting data from the sensor layer to a processing data unit. Transmissions are produced on wired or wireless channels. For this purpose, with wireless transmissions, a mobile device is often used as a relay to collect and retransmit the sensor's data to the processing unit.

- A processing layer: processing the sensor's data in order to classify the human activity.

Consequently, present user activity monitoring approaches have demonstrated to suffer the following drawbacks:

- They use specific hardware. These devices include the necessary sensor elements: accelerometers, gyroscopes, magnetometers, etc. [1] - [3].

- The sensor units have to be placed on specific body locations (hip, wrist, upper arm, etc.) and usually with specific tilt orientations to work properly. [4] [7].

- Sensor data is collected and locally transmitted using short-range wireless technologies, mostly Bluetooth, 
to communicate the data to a processing unit; or sometimes to communicate to a wireless device which will retransmit the data to the processing unit. The processing unit will finally process the information to classify the human activity. So more than one device is involved in the monitoring process.

In our proposal, a different approach is envisaged; its main features are summarized as follows:

- Using a conventional cell phone as the only device handled by the elderly or patient individual, as far as it includes some necessary sensors. Nevertheless, present cell phones are lately including these sensors, in particular: a tri-axial accelerometer, a magnetometer, an ambient light sensor, a GPS receiver and a Wifi receiver.

- Providing an easy way to configure the alerts to be triggered for each individual under surveillance.

- Monitoring any individual with a multiplatform access approach, basically allowing a web access monitoring but also having the possibility to receive those alerts on a cell phone or through software widgets.

- Lowering the overall monitoring system budget both for the end-users under surveillance and the supervisors as far as this proposal only uses conventional cell phones and computers.

- Defining a customized monitoring context instead of universal physical activity surveillance, including the history of the user daily habits. This way, achieving an implementation of the context awareness paradigm.

\section{SCOPE OF DEVELOPMENT}

Previous work by the authors on indoor location and user-movement classification was developed using the most elementary techniques on pattern recognition, basically the nearest neighbors technique, [1], [2], [3]. In a first step, one of the drawbacks on using the nearest neighbor technique is the need to calculate the Euclidean distance from any new input with all the recorded data inputs of the training set in order to classify the present input. It is not feasible when running this technique on the mobile phone instead of the server, or when considering an increasing number of recorded data inputs on the training phase in order to improve the pattern recognition accuracy.

Among the alternatives to the nearest neighbors technique to classify input data, the most relevant ones were: the neural networks and, in some cases, the support vector machine technique (SVM). In a first step, within the scope of the development, we took our previously developed prototypes on indoor location and movement recognition and we replaced the core pattern recognition modules with these two new techniques. Obtaining a more efficient implementation and consequently achieving the migration of the pattern recognition logic from the server to the mobile phone, the aim was to create, as much as possible, an autonomous application running completely on the mobile phone. Nevertheless, the neural networks were finally discarded due to its computational load when training the classifiers.

On a second step, the combination of the output data from the SVM classifiers (both the indoor location and movement estimation) together with the data provided by the GPS receiver and the ambient light sensor has been used to elaborate the user-monitoring service logic. This way the intended context awareness paradigm was materialized.

\section{SERVICE LOGIC}

The main device involved in this approach is the cell phone. As it is been said before, to implement a user activity monitoring system we need a sensor layer to recover the user activity and a processing layer to process the sensor's data to classify the user activity and trigger the corresponding alerts when necessary. Besides, we were referring to a transmission layer in order to transmit the sensor's data to the remote processing unit. In our case, the sensor and processing layers are included within the cell phone, so no transmission layer is necessary. In particular, the sensor layer involves:

- A tri-axial accelerometer and a magnetometer to monitor the user movements.

- A GPS receiver to monitor the user outdoor location.

- A Wi-fi receiver to monitor the user indoor location.

- An ambient light sensor to identify the darkness of the user location.

All the information provided by these sensors is filtered and processed by the same cell phone. Monitoring the user movements is implemented using pattern recognition techniques. As it is commonly the case on pattern recognition, there is a training phase to learn the system. From all the possibilities on pattern recognition techniques [7], [8], [9] we have chosen the SVM (support-vectormachine) classifiers as the best alternative to be implemented considering that the algorithm's execution had to be done in the cell phone.

For the outdoor location monitoring only periodic GPS requests were necessary to recover the intended data. The same applies for the ambient light sensor. Finally, to monitor the user indoor location we have followed the same procedure as for the movement's monitoring, using SVM classifiers we have trained the classifiers in an initial phase, to later on, use them when monitoring the user.

With this approach, we define a customized behavior pattern for each individual with the corresponding alert/reminder triggering criteria. We talk about reminders, not only alerts sent to external receivers, because the behavior pattern and also the included agenda with particular scheduled activities will trigger to the user the corresponding reminders when we detect, due to the sensors data, that the scheduled activity is not underway or that the recognized activity is out of the predicted behavior pattern or that a predicted activity due to the behavior pattern has not been detected. 
To define a customized behavior pattern or "usercontext" it was necessary to implement a multidimensional table including the following main entries:

- Time axis:

- A time table including the daily habits for the individual being monitored;

- An agenda with the scheduling for all outdoor visits to be paid.

- Location axis:

○ Outdoor: common outdoor locations to pay a visit and potential hazardous outdoor locations;

- Indoor: common sequence of rooms visited at home on an ordinary daily routine.

- Ambient light axis: Common ambient light levels at different indoor locations and times.

- Movements axis:

o Common series of user movements: walk, sitdown, stand-up, climb-up stairs, climb-down stairs, etc.;

- Identification of abnormal movements (falldowns).

The "user-context" is setup on the server and downloaded to the cell phone.

Alerts will be sent as e-mails, sms or pop-ups on installed computer widgets depending on the chosen configuration. Low-level alerts will be sent as far as the monitored individual is not following the predicted behavior pattern, all this without the user participation. Nevertheless there are some potential alerts, for example, when detecting that the user has fall down, where the monitoring application will ask the user, at least that this is a false alarm, and, if there is no response from the user to deactivate the alarm, this will be sent to the intended receivers including the emergency services, as it is shown on Figure 1 and 2.

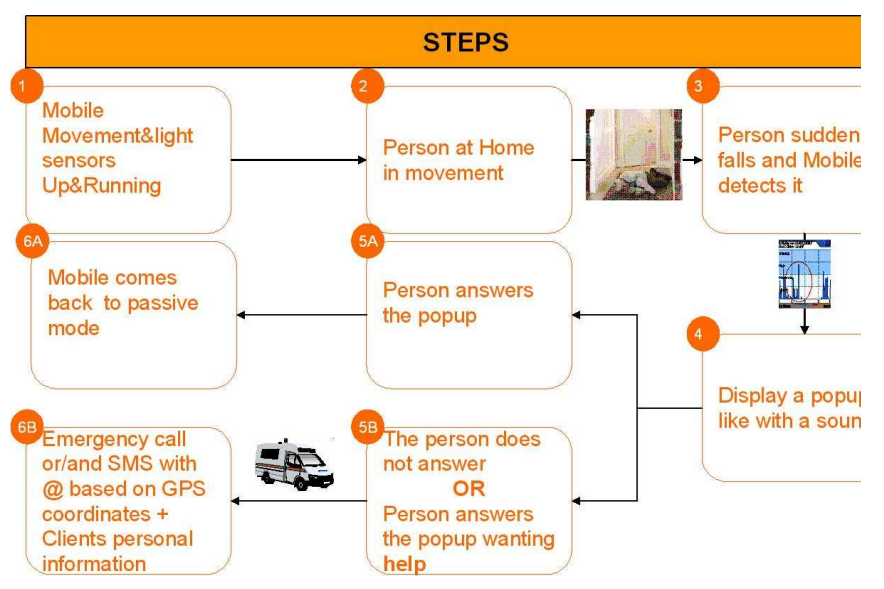

Figure 1. Fall down alert procedure

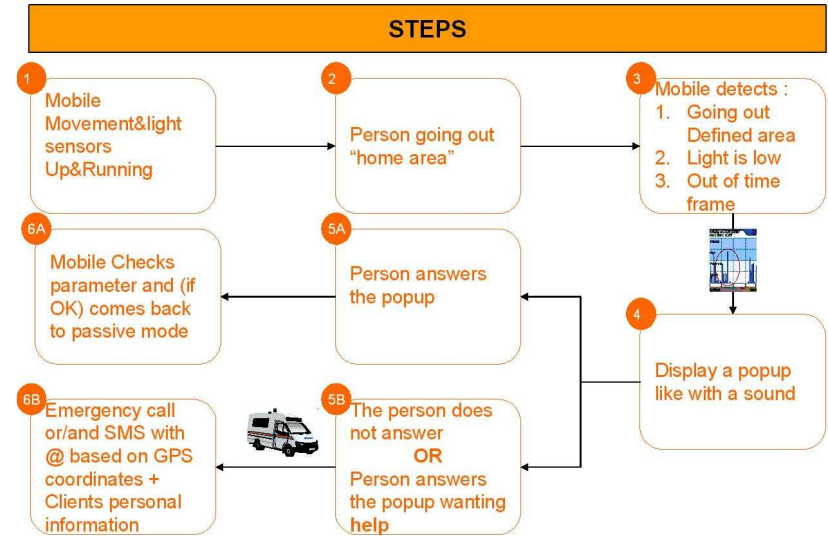

Figure 2. Outdoors alert procedure

\section{SERVICE SET UP}

A configuration platform is also involved in our approach. Basically, it sets up the alert triggering rules for each individual, including the alert delivery strategy with different options as we can see on Figure 3.

The so called server on Figure 3 only manages the users contact data to send alerts, the user's profiles, the alerts triggering thresholds and the alerts history of any individual. This way, on the cell phone a software application has been created to recover all the necessary data from the server and setup the monitoring system to be executed on this device.

To access the server a web application has been developed. After registering to the service, someone can fill up all the information related to the user to be monitored, just as an example on Figure 4 we can see the web page where the user will fill up the "habits" data.

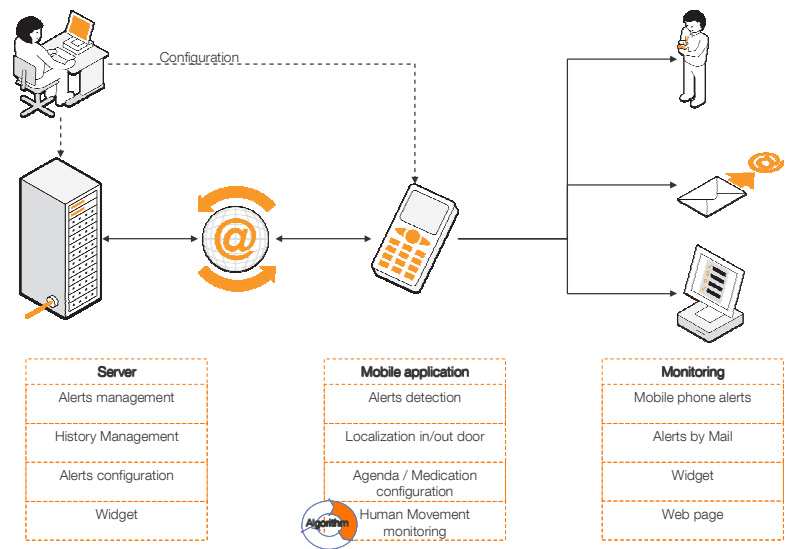

Figure 3. System architecture 


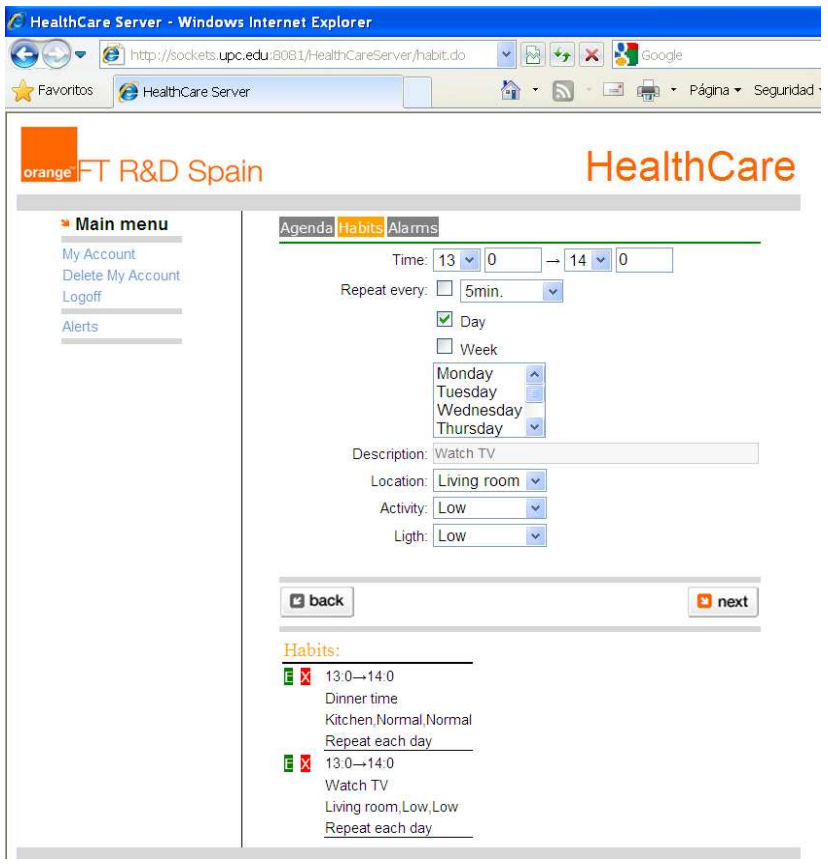

Figure 4. Filling up the "habits" data

The cell phone application is initiated as seen on Figure 5. From there on, after the login registration, it leads us to the user-data menu as seen on Figure 5. The user-data can be upload/download from the server. From there on we can go to the main menu.

The main menu of the phone application can be seen on Figure 6 . The first three buttons refer to the indoor location and user movements training. The fourth one refers to the user monitoring and the last one is used to recover the alert triggering rules introduced by the web application as it was explained before.

The indoor location training screen is seen on Figure 6, you must select the room where you are in, press the "start saving fingerprints" and move around the room for at least $5 \times 4$ measures of wi-fi signals before stopping the process.
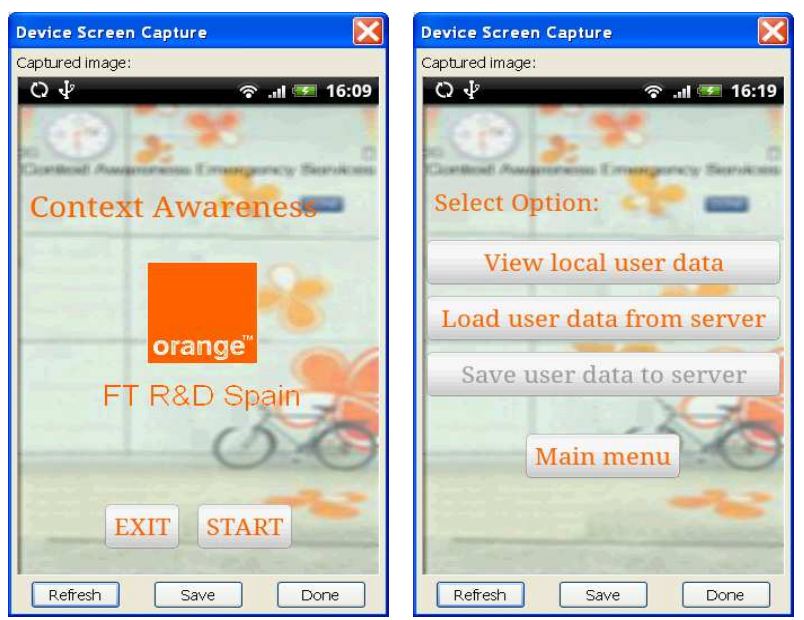

Figure 5. User-monitoring application

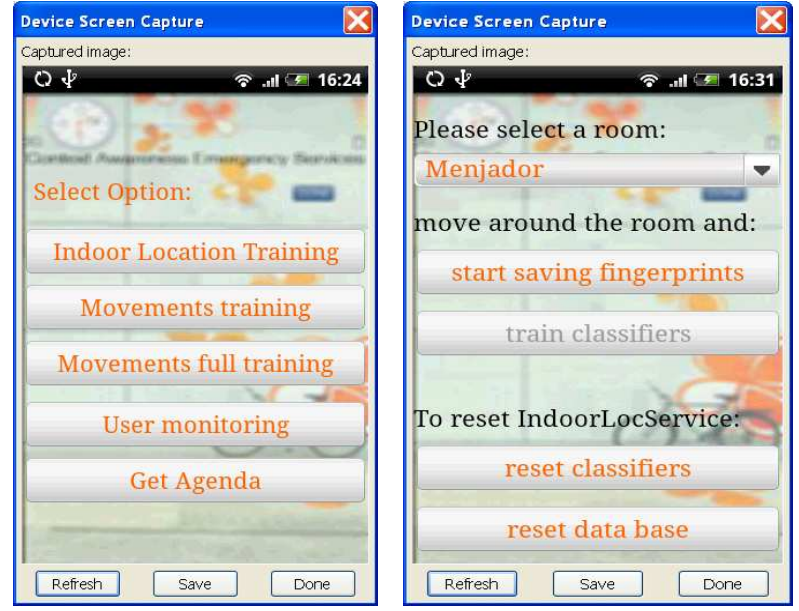

Figure 6. Main menu and indoor location training menu

In particular, from 5 single measures an average fingerprint measure is recorded, so we need at least 4 average fingerprint measures to train the system. Later on, you must stop the fingerprint saving, move to another room and repeat the same process, and so on in different rooms. At least measures from two different rooms are necessary before you can train the classifiers pressing the button "train classifiers". At any time you can reset the classifiers if you want to train them again or reset the complete data base losing all previously recorded wi-fi fingerprints in order to start from scratch the indoor location training.

The user movements' training will be made pressing the "Movements full training" from the main menu, see Figure 6 , nevertheless there is the opportunity to train the system for an individual movement pressing the "Movements training" button, on pressing this button you can select the individual movement and start training.

On Figure 7 you can see the movements' full training menu.

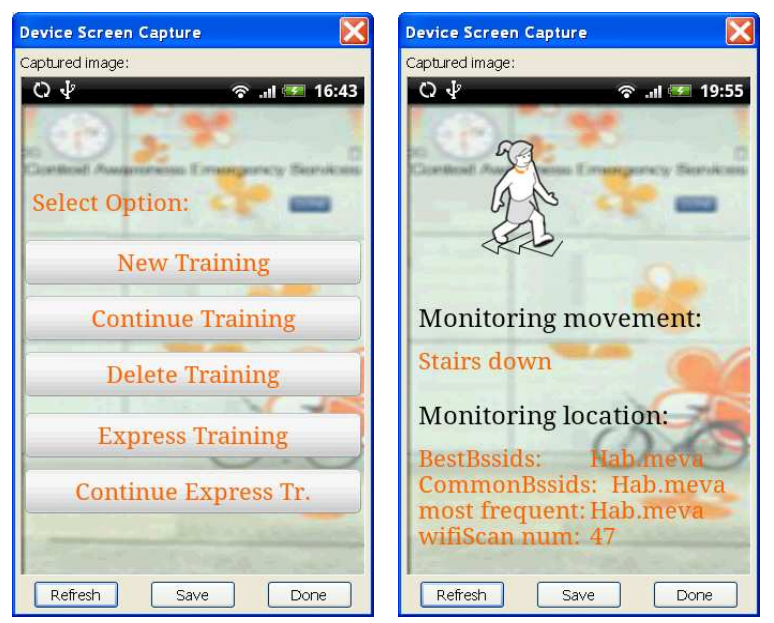

Figure 7. Movements training menu and user monitoring 
You can start the full training from the very beginning or continue a previous one where you left, you can delete all previously recorded data to start from scratch a new full movements' training; or finally, as it is stated on the last two buttons you can execute an express training from the very beginning or continue a previous one where you left. The express training is just a short version of the full training, although lest accurate, it is shorter to complete. On both: "new full training" or "new express training" you must follow a sequence of screens producing at each step, as you are said, the mentioned movements in order to record all the user's movements to complete the entire training process. Remember to, as much as possible; produce clear movements with the intended pause period just before and after each movement in order to increase the process accuracy.

Coming back to the main menu as on Figure 6, once the indoor location training and movements training are completed you can press the "user monitoring" button to visualize the monitoring process, see Figure 7 as a representative example of a screen capture while monitoring the user. Here different information will be displayed according to the present user activity and location.

Finally, according to the alert triggering rules, the corresponding alert, see Figure 8, will be triggered in order to confirm any detected abnormal situation, see previous screen on Figure 8. This way the user will cancel or confirm the alert, either with a voice command or pressing the corresponding button. If the alert is confirmed the necessary information will be supplied to the intended receivers, for example by sending a SMS message to the emergency contact phone number.

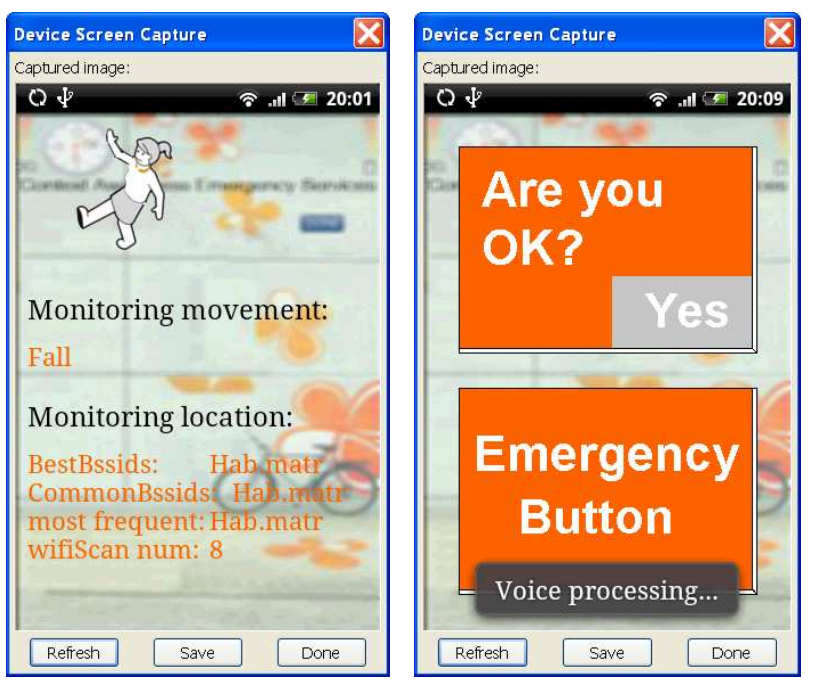

Figure 8. User monitoring (fall detection) and False alarm confirmation screen

\section{CONCLUSION}

In the present paper we have exposed the development of a real-time monitoring system of human activities using a single cell phone equipped with some particular sensors. No especial hardware is required, as far as a conventional cell phone will monitor the user, classify the user activities with respect to a customized behavior pattern and finally trigger the corresponding alerts or reminders when necessary. SVM classifiers have been used to recognize the user movements and indoor location, executing all the algorithms within the cell phone. A configuration platform has been developed to setup the customized behavior pattern to be downloaded to the cell phone, along with the different alternatives on alert delivery due to the multiplatform access proposal.

\section{ACKNOWLEDGMENT}

This work was partially supported by CICYT Grants TSI2007-66637-C02, TEC2006-04504 as well as European Project MIDAS (Multimodal Interfaces for Disabled and Ageing Society) in the ITEA programme (A strategic European programme for advanced R\&D in software for Software-intensive Systems and Services (SiS) ).

\section{REFERENCES}

[1] D.M. Karantonis, M.R. Narayanan, M. Mathie, N.H. Lovell,and B.G. Celler", "Implementation of a real-time human movement classifier using a triaxial accelerometer for ambulatory monitoring", IEEE Transactions on information tecnhology in biomedicine, vol. 10, no. 1, pp. 156-167, 2006.

[2] C. V. Bouten, K. T. Koekkoek, M. Verduin, R. Kodde, and J. D. Janssen", "A triaxial accelerometer and portable data processing unit for the assessment of daily physical activity," IEEE Transactions on Biomedical Engineering., vol. 44, no. 3, pp. 136-147, 1997.

[3] M. J. Mathie, A. C. F. Coster, N. H. Lovell, and B. G. Celler", "A pilot study of long term monitoring of human movements in the home using accelerometry," J. Telemed. Telecare, vol. 10, pp. 144$151,2004$.

[4] J. Fahrenberg, F. Foerster, M. Smeja, and W. Muller", "Assessment of posture and motion by multichannel piezoresistive accelerometer recordings," Psychophysiol., vol. 34, pp. 607-612, 1997.

[5] F. Foerster "and" J. Fahrenberg, "Motion pattern and posture: Correctly assessed by calibrated accelerometers," Behavior Research Methods, Instruments, \& Computers, vol. 32, pp. 450-457, 2000.

[6] P. H. Veltink, H. B. Bussmann, W. de Vries, W. L. Martens, and R. C. van Lummel", "Detection of static and dynamic activities using uniaxial accelerometers," IEEE Transactions on Rehabilitation Engineering, vol. 4, no. 4, pp. 375-385, 1996.

[7] Yoonseon Song, Seungchul Shin, Seunghwan Kim, Doheon Lee and Kwang. H. Lee", "Speed estimation from a tri-axial accelerometer using neural networks", 29th annual international conference of the IEEE EMBS, pp. 3224-3227 2007.

[8] Jhun-Ying Yang, Jeen-Shing Wang, and Yen-Ping Chen", "Using acceleration measurements for activity recognition: an effective learning algorithm for constructing neural classifiers", Pattern Recognition Letters, Elsevier, vol. 29, pp. 2213-2220, 2008.

[9] N. Ravi, N. Dandekar, P. Mysore, and M.L. Littman”, “Activity recognition from accelerometer data", American Association for Artificial Intelligence,

2005 . 